\title{
The journey of creating the first dedicated platform for robot-assisted (super)microsurgery in reconstructive surgery
}

\author{
Tom J. M. van Mulken ${ }^{1}$ (ID $\cdot$ Andrea M. J. Scharmga ${ }^{1} \cdot$ Rutger M. Schols $^{1} \cdot$ Raimondo Cau $^{2} \cdot$ Yasmine Jonis $^{1}$. \\ Shan S. Qiu ${ }^{1}$. René R. W. J. van der Hulst ${ }^{1}$
}

Received: 9 April 2019 / Accepted: 29 July 2019 / Published online: 6 September 2019

(C) The Author(s) 2019

\begin{abstract}
Incorporation of new technologies advances surgery in general. One example is robot assistance which can potentially overcome limitations in accessibility, dexterity, and ergonomics, thereby improving outcome of surgical procedures. To date, robot assistance has been used successfully in various fields of surgery. Also, in microsurgery robot assistance could offer great advantages as it requires great precision and meticulous technique. Currently available surgical robotic platforms are primarily designed for endoscopic procedures and lack the specific requirements for true microsurgery. Therefore, plastic surgeons of the Maastricht University Medical Center (Maastricht, the Netherlands) teamed up with engineers from the Eindhoven University of Technology (Eindhoven, the Netherlands) and developed the first dedicated robotic platform for (super)microsurgery, the MicroSure Robot (MSR).

Level of evidence: Level V, therapeutic study.
\end{abstract}

Keywords Robot assistance $\cdot$ Robotics $\cdot$ Reconstructive surgery $\cdot$ Microsurgery $\cdot$ Supermicrosurgery

\section{Introduction}

Surgical interventions are demanding processes, which require agile decision-making and meticulous execution. Especially in microsurgery qualities such as dexterity, maneuverability, steadiness, and technical quality are of utmost importance. The expertise needed to perform these procedures is acquired through extensive training and clinical experience. Over time, the instruments become extensions of the surgeons' hands. Notwithstanding, the surgical qualities required to perform microsurgery are limited to the individual surgeon and are susceptible to human fatigue, aging, and mindset. Undesired involuntary and inadvertent movement results in damage of tissue and substandard operation outcome [1]. Robot assistance can potentially

Tom J. M. van Mulken tom.van.mulken@mumc.nl

1 Department of Plastic, Reconstructive and Hand Surgery, Maastricht University Medical Center, P. Debyelaan 25, PO 5800, 6202 AZ Maastricht, the Netherlands

2 Department of Medical Robotic Technologies, Eindhoven University of Technology, Eindhoven, the Netherlands overcome the aforementioned issues. Robot assistance provides filtering of tremor, enhances dexterity, and may enable more surgeons to perform (super)microsurgery. Incorporation of robotics in microsurgery would increase the quality of current microsurgical and supermicrosurgical procedures and create new possibilities in the field of (super)microsurgery. This master class article aims to describe the challenging journey of developing a dedicated robotic platform for (super)microsurgery - from inception to first introduction in a clinical setting.

\section{Inception of the idea}

From the 1970s onward, ideas have led to the development of the first (tele)robots in surgery [2]. Whether it was to operate on astronauts in space or to assist and function as supporting manipulators, the ideas have evolved into a new era of robot-assisted surgery. The first Food and Drug Administration-approved robot was the da Vinci Surgical System (Intuitive Surgical, Inc.) which has been used successfully in various surgical specialties, such as cardiac surgery, neurosurgery, and urology [3-6]. This robotic system was designed for endoscopic surgery and has been successfully used for endoscopic 
harvesting and in setting of flaps [7, 8]. However, the system showed significant limitations when used for microsurgical anastomoses in free flap reconstruction [9-13]. The actual idea of creating a dedicated robotic platform for microsurgery arose when the da Vinci system was used during the first robot-assisted microsurgical anastomosis in reconstructive microsurgery in 2007 [13]. In a mini-muscle TRAM-flap breast reconstruction, the arterial anastomosis on the internal mammary artery was performed using robot assistance. Although the anastomosis was successfully completed, the authors concluded that the system had many limitations for application in microsurgery. The limited optic magnification and the bulky and powerful instruments of the system were significant drawbacks for use in true microsurgery.

\section{Design concept phase}

Based on this first experience with robot-assisted microsurgery, a Dutch collaboration between microsurgeons of the Maastricht University Medical Center (MUMC+, Maastricht, the Netherlands) and engineers of the Eindhoven University of Technology (TU/e, Eindhoven, the Netherlands) started the development of a dedicated robotic platform for (super)microsurgery: the Microsure Robot (MSR) [14]. Analyses of conventional methodologies and available alternative solutions were taken into account as a guideline for the appropriate requirements of the MSR. The development of the MSR was primarily focused on improving the surgeons' performance by filtering tremor and scaling movements, while at the same time being easily integrated into the existing workflow and infrastructure in the operating theatre. Complementary to this, but not least important, were safety, ease-of-use, and cost-effectiveness.

\section{Proof-of-concept phase}

Consequently, the design concept was transformed into a proof-of-concept. The system has previously been described in previous publications $[15,16]$. This proof-of-concept consisted of (Figs. 1 and 2):

1. Suspension ring, that can be mounted onto the surgical table or the microscope

2. User interface, forceps-like joysticks which copy the surgeons' movement to a micro-instrument held by the device (robotic arms)

3. Robotic arms, equipped with genuine microsurgical instruments which effectuate the surgeons' movement

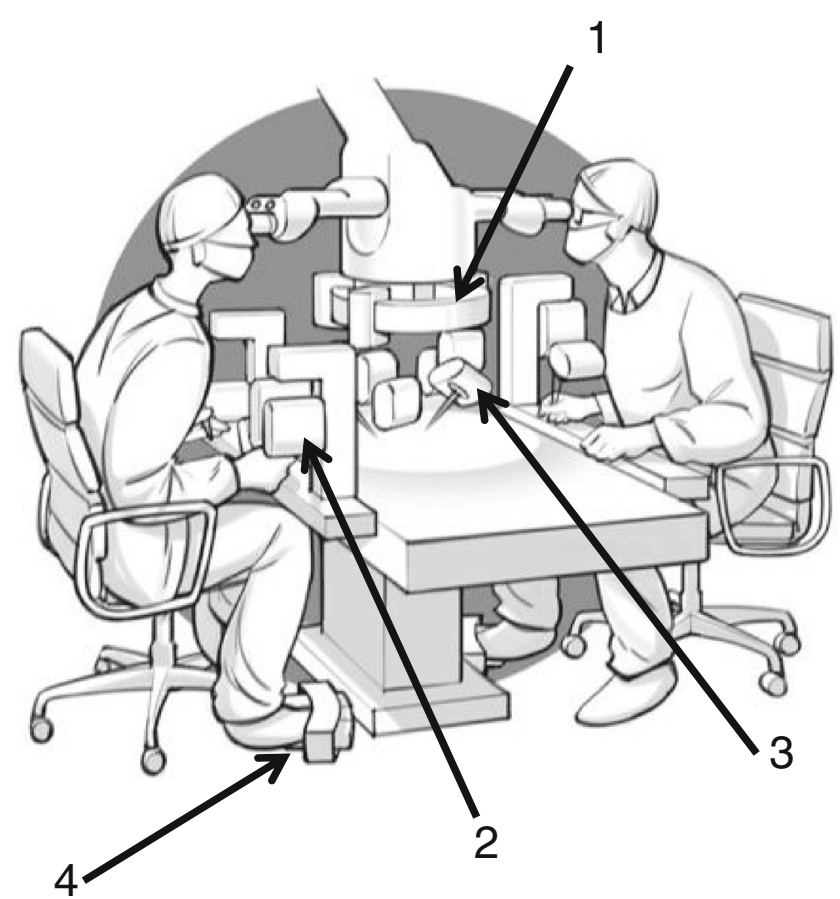

Fig. 1 Setup of the MSR. 1, suspension ring; 2, user interface; 3, robotic arms; 4 foot pedals

4. Food pedals, controlling the motion scaling

5. The device software, scaling down motion and filtering out tremor

The setup of the MSR allows the surgeon to freely use a conventional surgical microscope in combination with the device. The unchanged proximity of the surgeon to the patient allows the surgeon to get a direct view and access to the surgical field at all times if necessary and the robotic arms can easily be moved aside to continue the microsurgical

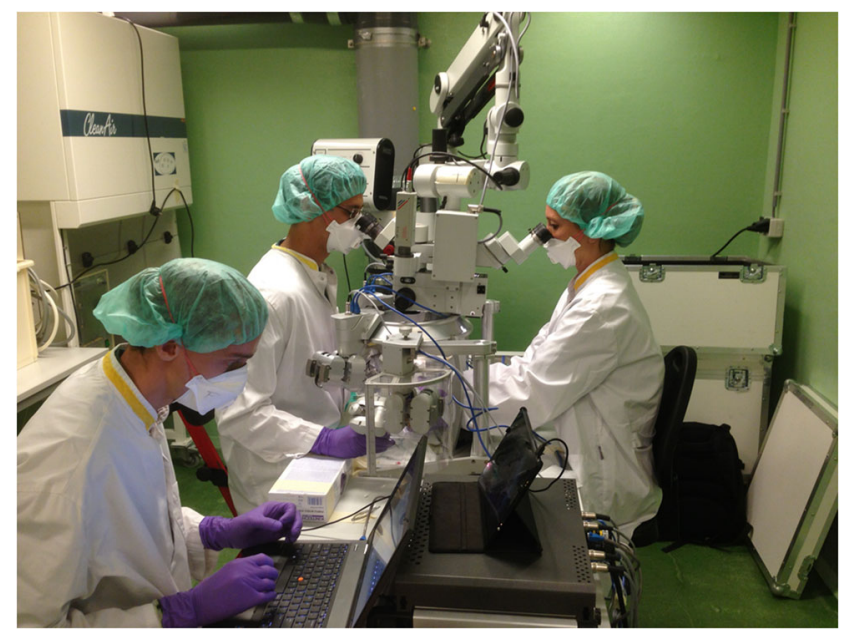

Fig. 2 Initial setup of the proof of concept robot MSR [16] 
procedure manually. This flexibility in switching between robot-assisted and conventional microsurgery during the procedure allows hybrid operations to be performed. This reduces total surgical time by enabling high-precision robot assistance only for phases of the operation where this is necessary.

The system is based on a modular design, permitting it to be used in different configurations depending on the level of required robot assistance. Up to four robotic arms can be used simultaneously, allowing one or two operating surgeons to control them by means of the user interface.

During the surgical procedure, the robot is entirely covered in sterile draping, except for the sterilized adapters that can be loaded with genuine microsurgical instruments.

This feature of the robotic platform allows the surgeon to use his or her preferred original instruments and skill set yet having the additional benefits of tremor filtration and motion scaling. The small footprint of the MSR creates a virtually unchanged setup and workflow in the operating theatre with respect to the conventional approach.

\section{Pre-clinical phase}

The first preclinical experience of the MSR was previously shared in a study investigating its performance in microsurgical anastomosis on 2-mm silicone vessels (Fig. 3) [15]. Three participants with different levels of microsurgical training (an experienced microsurgeon, a resident in plastic surgery, and a medical student) each completed ten anastomoses by the conventional manual method and ten anastomoses using the MSR. All anastomoses were video recorded and photographed through a microscope. Time to perform the anastomosis was logged (from grasping the needle to cutting the last suture). Videos and photos were assessed by four experienced surgeons blinded to the subjects' identity, using a

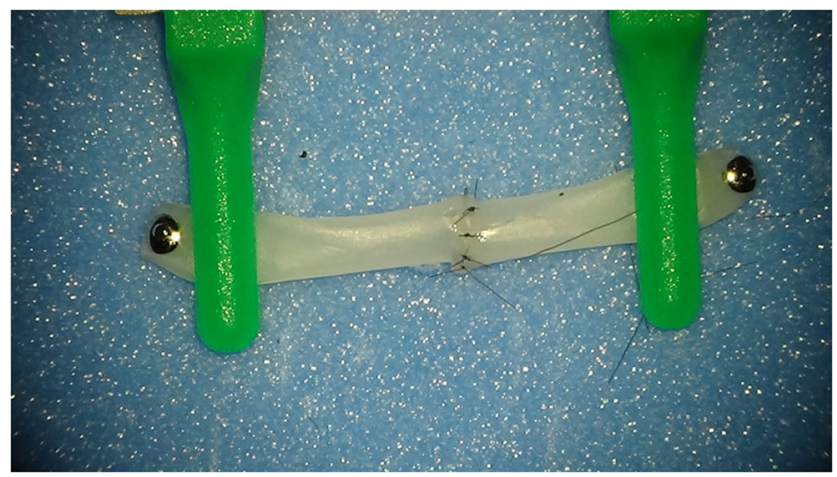

Fig. 3 Robot-assisted anastomosis on 2-mm diameter silicone vessel [15] modified version of the Structured Assessment of Microsurgical Skills (SAMS) (for the videos) [17], and spacing of sutures and quality of knots were analyzed (photos). Results showed that the total time to perform the anastomoses decreased from 32 to 13 min (manual procedures), and from 119 to $35 \mathrm{~min}$ (robot-assisted procedures). Improvement was also observed in the overall performance (manual 2.8 to 3.6 and robot 2.3 to 3.0 ) and indicative skill domains (manual 2.6 to 3.7 and robot 2.3 to 3.1 ) of the modified SAMS.

This study showed promising results of the feasibility of performing anastomoses using the MSR on silicone vessels in a pre-clinical setting. Although time to perform the anastomosis was longer using robot assistance, and quality assessed by the modified SAMS was lower compared with the conventional anastomoses, steeper learning curves were observed for the robot-assisted approach in both time and surgical skills.

Encouraged by the aforementioned study, we progressed in evaluating the feasibility of the MSR on anastomoses in an in vivo rat model (Fig. 4) [16]. The abdominal aorta and femoral artery in rats show good resemblance with the vessels used in (super)microsurgical free flap anastomosis [18]. The diameter of the aorta was 1.8 to $2.4 \mathrm{~mm}$ and the diameter of the femoral artery was 0.7 to $0.8 \mathrm{~mm}$. In this study, one microsurgeon performed all anastomoses. Time to perform anastomosis was recorded, and quality of preparation, piercing, knot tying, and additional actions were analyzed. Furthermore, any adverse events were logged. In total, seven anastomoses were performed using the MSR (three anastomoses of the abdominal aorta and four of the femoral artery). As a reference, two additional anastomoses were performed using the conventional manual method. Time to perform an

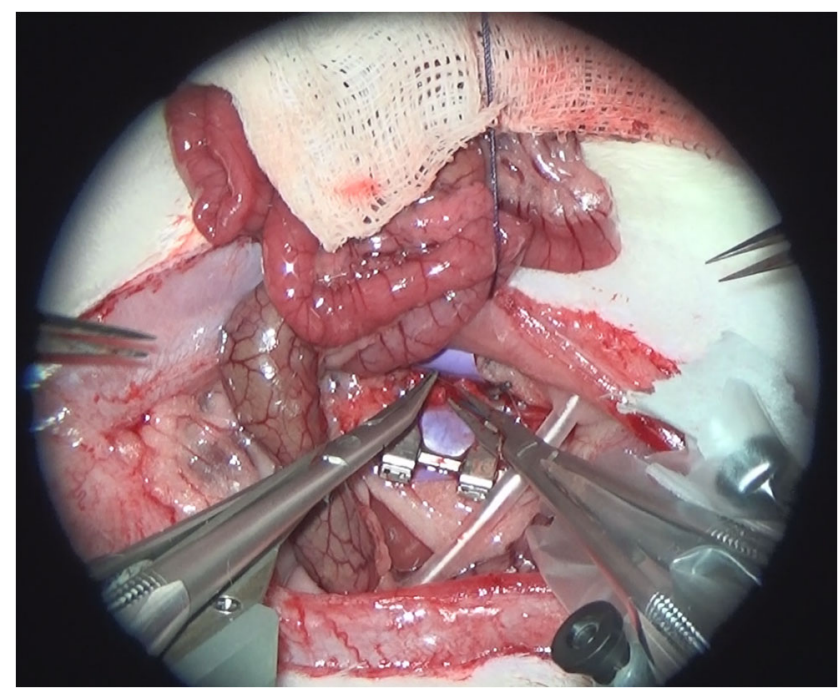

Fig. 4 Robot-assisted anastomosis of abdominal aorta in a rat model [16] 
anastomosis was longer using the MSR, for both aorta and femoral artery (mean (range), 69 (53-87) minutes and 27 (26-29) minutes, respectively), as compared with the two manual anastomoses (19 and $12 \mathrm{~min}$ ). All abdominal aorta anastomoses and three out of four femoral artery anastomoses were patent using the MSR. During the robotic procedures, nine adverse events occurred, including a robotic arm reset $(n=3)$, a system reset $(n=2)$, and a change of instruments $(n=3)$. The results of this in vivo rat model underlined the previous conclusion that the MSR is able to complete a microsurgical anastomosis. Again, time to perform the anastomosis was longer compared with the manual method. However, the results were solid enough to enter a next phase of the MSR.

\section{Clinical evaluation and future perspectives}

Based on insights gained during the preclinical phase, a new and improved version of the MSR, Microsure's MUSA (Microsure, Eindhoven, Tthe Netherlands) was built for evaluation in a clinical setting (Fig. 5a and b). This study is still in progress and data will be made available in the near future.

Evolution in technology has changed the possibilities in the medical field. Robot assistance in microsurgery can overcome human limitations in precision and ergonomics. Furthermore, additional benefits of robot assistance can be explored in the future as well, namely;

\section{Tactile feedback}

Conventional (super)microsurgery is performed without tactile feedback as forces are too low to rely on [19]. Incorporation of enhanced tactile feedback in robot assistance would enable the surgeon to feel extremely delicate forces and therefore enhance tissue handling and precision which could reduce adverse events and improve patient outcome in (super)microsurgery.

\section{Microsurgical training}

The training of microsurgical skills is currently based on subjective observations of other trained microsurgeons. Parameters such as completion time, path length, depth perception, speed, smoothness, efficiency, and bi-manual dexterity are evaluated subjectively. Objective measurement of motion and forces can improve the evaluation of surgical performance and enhance microsurgical training and clinical care. Future surgeons will need to be trained in both conventional and robot-assisted microsurgery techniques to be capable of dealing with the wide variety of surgical problems that they will encounter throughout their career. Therefore, both robot-assisted and conventional microsurgical experience should be incorporated into surgical training [19].

\section{Intra-operative imaging techniques}

Robot assistance in microsurgery can be further enhanced by incorporating novel optical imaging techniques such as three-dimensional imaging, highresolution stereotactic operation, spectral imaging, and real-time navigation. Intra-operative imaging such as near-infrared fluorescence imaging particularly offers anatomical guidance, such as better vascular and lymph and nerve visualization, relevant to (super)microsurgery [20].
Fig. 5 Microsure's MUSA robot. New generation of the Microsure robot
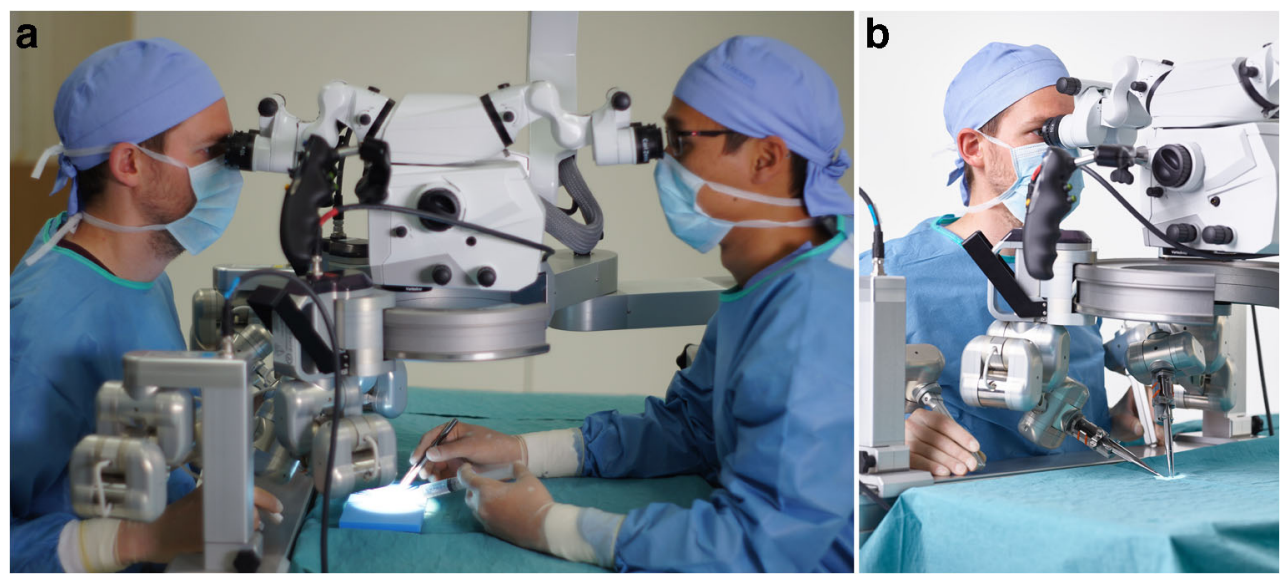


\section{Machine learning}

As a subset of artificial intelligence, machine learning uses decision-making computer algorithms to understand and respond to specific data [21]. Already applied in a wide spectrum of robotic applications, machine learning has only been considered for robotic surgery recently [22]. The development of these cognitive surgical robots endows robotic systems with the ability of self-learning and cognitive skills, thereby enabling automation, which can improve and standardize our microsurgical techniques [22]. Improving quality and efficiency in microsurgery extends beyond the surgical procedure. Combining patient-related data with robot registration can provide better understanding of the consequences of decisions and interventions during surgery, ultimately leading to better patient care.

\section{Conclusions}

The initial idea of developing a robot that is dedicated to (super)microsurgery resulted in a platform that is feasible to perform (super)microsurgical anastomosis. Robot assistance enables tremor filtration and enhances dexterity and can train more surgeons for (super)microsurgery. It has the potential to increase the quality of current microsurgical and supermicrosurgical procedures and create new possibilities in the field of (super)microsurgery. The increasing importance of quality, patient safety, and cost efficiency will propel the development of new robotic technology for microsurgery. Further research and clinical testing are needed to use robot assistance to its full potential and open new possibilities in (super)microsurgery.

Funding This study did not receive any specific grant from funding agencies in the public, commercial and not-for-profit sectors.

\section{Compliance with ethical standards}

Conflict of interest Tom van Mulken is Chief Medical Officer of the spin off company MicroSure and shareholder in the company. René van der Hulst is shareholder of MicroSure. Raimondo Cau is Chief Technical Officer and shareholder of MicroSure. The other authors declare that they have no conflict of interest.

Ethical approval Ethical approval for the pre-clinical study on rats was obtained from the Maastricht University Institutional Review Board for Animal Use in Research (Maastricht, the Netherlands; project reference: DEC 2014-078)
Open Access This article is distributed under the terms of the Creative Commons Attribution 4.0 International License (http:// creativecommons.org/licenses/by/4.0/), which permits unrestricted use, distribution, and reproduction in any medium, provided you give appropriate credit to the original author(s) and the source, provide a link to the Creative Commons license, and indicate if changes were made.

\section{References}

1. Riviere CN, Khosla PK (eds) (1998) Active handheld instrument for error compensation in microsurgery. Intelligent systems and advanced manufacturing. SPIE

2. Takacs A, Nagy DA, Rudas IJ, Haidegger T (2016) Origins of surgical robotics: from space to the operating room. Acta Polytech Hung 13(1):13-30

3. Loulmet D, Carpentier A, d'Attellis N, Berrebi A, Cardon C, Ponzio O et al (1999) Endoscopic coronary artery bypass grafting with the aid of robotic assisted instruments. J Thorac Cardiovasc Surg 118(1):4-10

4. Boyd WD, Desai ND, Kiaii B, Rayman R, Menkis AH, McKenzie FN et al (2000) A comparison of robot-assisted versus manually constructed endoscopic coronary anastomosis. Ann Thorac Surg 70(3):839-842 discussion 42-3

5. Latif MJ, Afthinos JN, Connery CP, Perin N, Bhora FY, Chwajol M et al (2008) Robotic intercostal nerve graft for reversal of thoracic sympathectomy: a large animal feasibility model. Int J Med Robot 4(3):258-262

6. Dickey RM, Pastuszak AW, Hakky TS, Chandrashekar A, Ramasamy R, Lipshultz LI (2015) The evolution of vasectomy reversal. Curr Urol Rep 16(6):40

7. Ibrahim AE, Sarhane KA, Pederson JC, Selber JC (2014) Robotic harvest of the rectus abdominis muscle: principles and clinical applications. Semin Plast Surg 28(1):26-31

8. Selber JC (2011) Robotic latissimus dorsi muscle harvest. Plast Reconstr Surg 128(2):88e-90e

9. Katz RD, Rosson GD, Taylor JA, Singh NK (2005) Probotics in microsurgery: use of a surgical robot to perform a free flap in a pig. Microsurgery 25(7):566-569

10. Katz RD, Taylor JA, Rosson GD, Brown PR, Singh NK (2006) Robotics in plastic and reconstructive surgery: use of a telemanipulator slave robot to perform microvascular anastomoses. J Reconstr Microsurg 22(1):53-57

11. Selber JC (2010) Transoral robotic reconstruction of oropharyngeal defects: a case series. Plast Reconstr Surg 126(6):1978-1987

12. Taleb C, Nectoux E, Liverneaux PA (2008) Telemicrosurgery: a feasibility study in a rat model. Chir Main 27(2-3):104-108

13. van der Hulst R, Sawor J, Bouvy N (2007) Microvascular anastomosis: is there a role for robotic surgery? J Plast Reconstr Aesthet Surg 60(1):101-102

14. Cau R (2014) Design and realization of a master-slave system for reconstructive surgery, Eindhoven

15. van Mulken TJM, Boymans CAEM, Schols RM, Cau R, Schoenmakers FBF, Hoekstra LT et al (2018) Preclinical experience using a new robotic system created for microsurgery. Plast Reconstr Surg 142(5):1367-1376

16. van Mulken TJM, Schols RM, Qiu SS, Brouwers K, Hoekstra LT, Booi DI et al (2018) Robotic (super) microsurgery: feasibility of a new master-slave platform in an in vivo animal model and future directions. J Surg Oncol 118(5):826-831 
17. Alrasheed T, Liu J, Hanasono MM, Butler CE, Selber JC (2014) Robotic microsurgery: validating an assessment tool and plotting the learning curve. Plast Reconstr Surg 134(4):794-803

18. Shurey S, Akelina Y, Legagneux J, Malzone G, Jiga L, Ghanem AM (2014) The rat model in microsurgery education: classical exercises and new horizons. Arch Plast Surg 41(3):201-208

19. Tan YPA, Liverneaux P, Wong JKF (2018) Current limitations of surgical robotics in reconstructive plastic microsurgery. Front Surg $5: 22$

20. Cornelissen AJM, van Mulken TJM, Graupner C, Qiu SS, Keuter XHA, van der Hulst R et al (2018) Near-infrared fluorescence image-guidance in plastic surgery: a systematic review. Eur J Plast Surg 41(3):269-278
21. Aruni G, Amit G, Dasgupta P (2018) New surgical robots on the horizon and the potential role of artificial intelligence. Investig Clin Urol 59(4):221-222

22. Kassahun Y, Yu B, Tibebu AT, Stoyanov D, Giannarou S, Metzen JH et al (2016) Surgical robotics beyond enhanced dexterity instrumentation: a survey of machine learning techniques and their role in intelligent and autonomous surgical actions. Int J Comput Assist Radiol Surg 11(4):553-568

Publisher's note Springer Nature remains neutral with regard to jurisdictional claims in published maps and institutional affiliations. 